\title{
Functional properties and sequence variation of HTLV-1 p13
}

\author{
Maria Omsland ${ }^{1}$, Micol Silic-Benussi ${ }^{2}$, Ramona Moles ${ }^{1}$, Sarkis Sarkis ${ }^{1}$, Damian F. J. Purcell ${ }^{3}$, David Yurick ${ }^{3}$, \\ Georges Khoury ${ }^{3,6}$, Donna M. D'Agostino ${ }^{4}$, Vincenzo Ciminale ${ }^{2,5}$ and Genoveffa Franchini ${ }^{*}$
}

\begin{abstract}
Human T cell leukemia virus type-1 (HTLV-1) was the first retrovirus found to cause cancer in humans, but the mechanisms that drive the development of leukemia and other diseases associated with HTLV-1 infection remain to be fully understood. This review describes the functional properties of p13, an 87-amino acid protein coded by HTLV-1 open reading frame II (orf-II). p13 is mainly localized in the inner membrane of the mitochondria, where it induces potassium $\left(\mathrm{K}^{+}\right)$influx and reactive oxygen species (ROS) production, which can trigger either proliferation or apoptosis, depending on the ROS setpoint of the cell. Recent evidence indicates that p13 may influence the cell's innate immune response to viral infection and the infected cell phenotype. Association of the HTLV-1 transcriptional activator, Tax, with p13 increases p13's stability, leads to its partial co-localization with Tax in nuclear speckles, and reduces the ability of Tax to interact with the transcription cofactor CBP/p300. Comparison of p13 sequences isolated from HTLV-1-infected individuals revealed a small number of amino acid variations in the domains controlling the subcellular localization of the protein. Disruptive mutations of p13 were found in samples obtained from asymptomatic patients with low proviral load. p13 sequences of HTLV-1 subtype C isolates from indigenous Australian patients showed a high degree of identity among each other, with all samples containing a pattern of 5 amino acids that distinguished them from other subtypes. Further characterization of p13's functional properties and sequence variants may lead to a deeper understanding of the impact of p13 as a contributor to the clinical manifestations of HTLV-1 infection.
\end{abstract}

Keywords: Human T cell leukemia virus type-1, HTLV-1, HTLV-1C, p13, Mitochondria, Cell death

\section{Background}

Human T cell leukemia virus type 1 (HTLV-1) is a retrovirus that causes an aggressive neoplasm of mature $\mathrm{CD} 4^{+}$ $\mathrm{T}$ cells called adult $\mathrm{T}$ cell leukemia (ATL), as well as a variety of inflammatory diseases including uveitis, infectious dermatitis, Sjögren's syndrome, and HTLV-1-associated myelopathy/tropical spastic paraparesis (HAM/ TSP) [1-8]. Although at least 5-10 million people are infected with HTLV-1 worldwide, only a small percentage

\footnotetext{
*Correspondence: franchig@mail.nih.gov

${ }^{1}$ Animal Models and Retroviral Vaccines Section, Vaccine Branch, Center

for Cancer Research, National Cancer Institute, National Institutes of Health, Bethesda, MD, USA

Full list of author information is available at the end of the article
}

will develop clinically relevant symptoms [9]. The factors that determine the clinical outcome of HTLV-1 infection are not fully understood, but studies aimed at investigating the viral determinant of HTLV-1 pathogenicity have underscored the oncogenic potential of Tax and HBZ [10].

Despite having a relatively small genome of approximately 9000 nt [11], HTLV-1 expresses multiple gene products through the transcription of both strands of its proviral genome, complex mRNA splicing, and ribosomal frameshifting, resulting in the production of protease and polymerase enzymes, the structural Gag and Env proteins, and the non-structural proteins Tax, Rex, p21Rex, p30, p13, p12/p8, and HBZ. This review describes the biological properties of $\mathrm{p} 13$, a small protein encoded by 
the second open reading frame of the $\mathrm{X}$ region (orf-II) [12]. As we explore here, recent studies have revealed interesting variations in p13 sequences isolated from different patients infected with HTLV-1 $[13,14]$ that may possibly be relevant to the life cycle and pathogenic properties of HTLV-1.

\section{Expression and intracellular localization of $\mathrm{p} 13$}

Early studies of orf-II revealed that it codes for two proteins: p30, a 241-residue nuclear/nucleolar protein expressed from a doubly-spliced mRNA, and p13, an 87 -residue protein coded by a singly-spliced mRNA corresponding to the carboxy-terminal portion of $\mathrm{p} 30[12$, $15,16]$. Analyses of the expression kinetics of the viral transcripts in infected cells showed that the p13 and p30 mRNAs accumulate late in the replication cycle, together with mRNAs encoding structural proteins $[17,18]$. Initial studies carried out in a HeLa-derived cell line transiently transfected with p13 expression plasmids indicated that the protein accumulated in punctate structures located in the cytosol and perinuclear area, and in the nucleus but not nucleoli [15]. Subsequent co-localization analysis with compartment markers revealed that the punctate structures containing p13 were in fact mitochondria [19]. The results of mutational analyses and assays with GFP-tagged portions of p13 led to the identification of the minimal mitochondrial targeting signal (MTS) that is necessary and sufficient to determine the protein's mitochondrial accumulation (Fig. 1) [19]. Circular dichroism analysis showed that the p13 MTS folds into an amphipathic alpha helical structure containing four arginines [20]. Unlike canonical MTS, the p13 MTS differs is not located at the amino terminus of the protein, it is not cleaved upon import, and it does not require the presence of the four arginines for mitochondrial localization [20]. The mitochondrial localization of p13 was confirmed by confocal microscopy analysis and a combination of electron microscopy and biochemical fractionation studies, which revealed that p13 is mainly inserted in the inner mitochondrial membrane [20]. Confocal microscopy analysis also confirmed mitochondrial localization in transfected primary rat embryo fibroblasts and the $\mathrm{T}$ cell acute lymphoblastic leukemia (T-ALL) cell line Jurkat [21].

The MTS of p13 acts as a dominant targeting signal that is necessary for the mitochondrial accumulation of $\mathrm{p} 13$, and is sufficient to direct the mitochondrial accumulation of heterologous proteins such as GFP [19]. The $13-\mathrm{kDa}$ size of $\mathrm{p} 13$ is well below the cut-off of the nuclear pore, suggesting that the protein should be able to freely diffuse in and out of the nucleus. As depicted in Fig. 1, p13 is believed to contain a nuclear localization signal (NLS) positioned after its MTS. The existence of this NLS was inferred from observations from a series of deletion mutants of p30 fused to GFP [22], and further analysis is required to verify its impact on p13's intracellular compartmentalization. A study by Andresen et al. showed that p13 becomes more stable when co-expressed with Tax, that it is modified by ubiquitination, and that a small fraction of p13 is localized to nuclear speckles containing Tax and SC35 (Fig. 2) [23]. Interestingly, the nuclear localization of $\mathrm{p} 13$ was more prominent when the protein was fused to green fluorescent protein (GFP) or to the hemagglutinin (HA) epitope tag (unpublished data). The nuclear accumulation of p13-GFP also appeared to be proportional to the expression levels of the protein (Fig. 2). These findings suggest that p13 may accumulate in the nucleus when a certain concentration threshold is reached, which might be favored by Tax or the presence of tags such as ubiquitin.

\section{Functional properties of $\mathrm{p} 13$ Effects of $p 13$ on $\mathrm{K}^{+}$flux}

Studies carried out using synthetic p13 and purified mitochondria showed that the protein induces a potassium influx through the inner mitochondrial membrane $(\Delta \psi)[20,24]$. The entry of $\mathrm{K}^{+}$increases the activity of the electron transport chain (ETC), which extrudes a higher

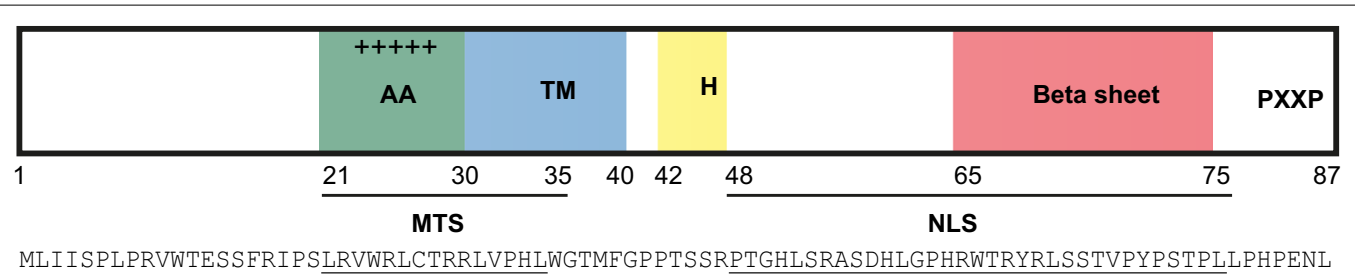

Fig. 1 p13 domain structure. Schematic representation of the domain structure of p13. AA indicates the amphipathic a-helix overlapping with the mitochondrial targeting signal (MTS, amino acids 21-35) and +++ indicates the four arginines present in the MTS. The transmembrane region (TM) includes amino acids 30-40. A region with a high flexibility score $(H)$ spans amino acids $42-48$. A predicted $\beta$-sheet structure spans amino acids 65-75. The proline-rich C-terminus contains two overlapping P-x-x-P motifs implicated in interactions with SH3 domain-containing proteins. A putative cryptic nuclear localization sequence (NLS) is mapped to a region spanning residues 43-80. This figure was adapted from Figure 1 in [87] 

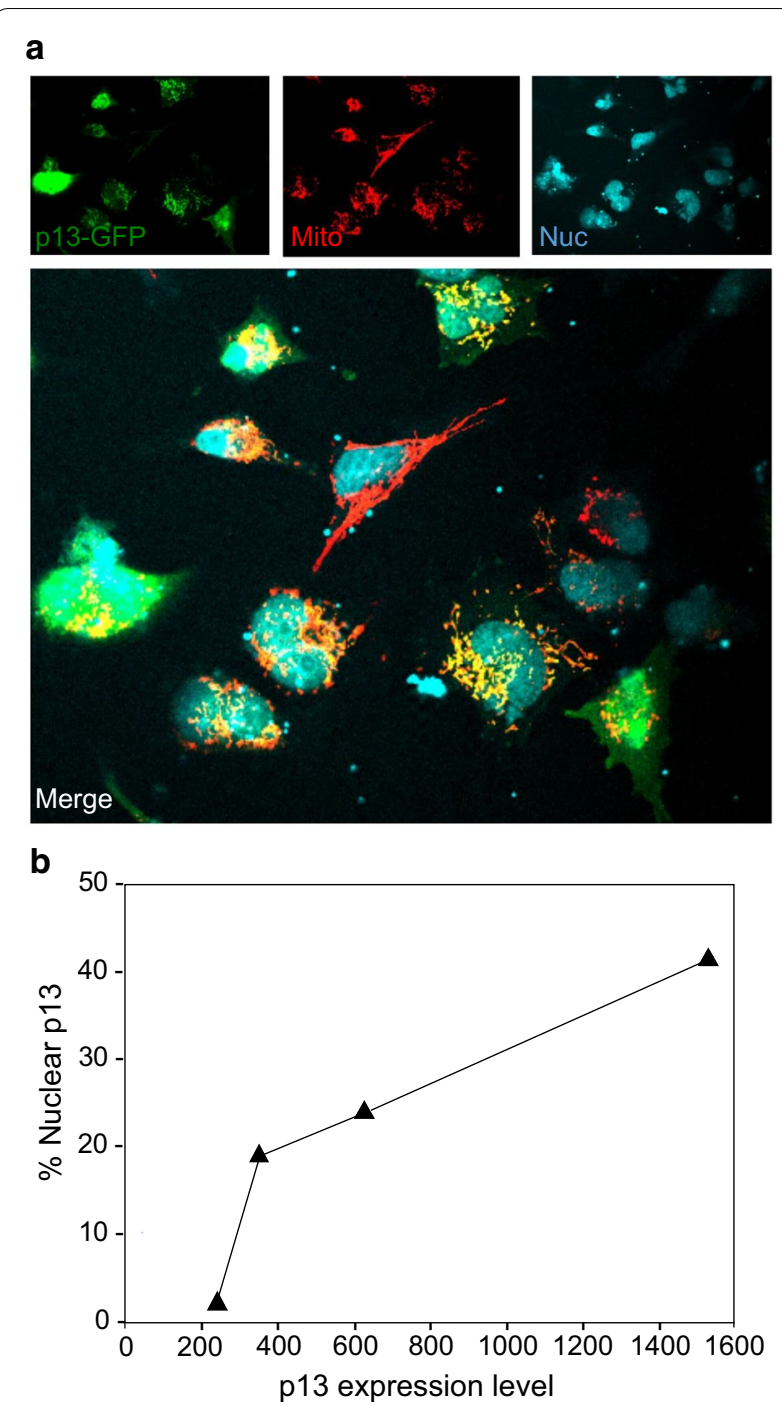

Fig. 2 Intracellular localization of p13-GFP. a Confocal microscopy analysis of HeLa cells transfected with a p13-GFP-expressing plasmid and labelled with an antibody recognizing the mitochondrial protein HSP60 (Santa Cruz Biotechnology) and an Alexa-546-conjugated secondary antibody (Life Technologies). Nuclei were visualized using Vibrant DyeCycle Ruby (Life Technologies). Nuclei, mitochondria, and p13 are shown in blue, red, and green, respectively. b Quantitative analysis of the percentage of p13-GFP signal detected in nucleus in relation to the total p13-GFP signal measured in the same cell

number of $\mathrm{H}^{+}$and thus balances the entry of $\mathrm{K}^{+}$positive charges. Although this ramping up of the ETC maintains the potential of the mitochondrial membrane, it also favors the production of reactive oxygen species (ROS). Increased ROS may trigger the opening of the mitochondrial permeability transition pore (PTP), an event that can lead to cell death [25] (Fig. 3).

Cells expressing p13 have fragmented mitochondria [20]. The effect of p13 in both isolated mitochondria and in cells is dose-dependent: When p13 is low, the entry of $\mathrm{K}^{+}$is counterbalanced by increased ETC activity. At higher levels of p13, cells show mitochondrial depolarization and substantial fragmentation. Although the four arginines in the amphipathic alpha helix are not essential for mitochondrial targeting, they are required to induce the inflow of $\mathrm{K}^{+}$into mitochondria and for mitochondrial fragmentation [20, 24].

\section{p13 and mitochondrial ROS}

ROS are powerful second messengers that control multiple signal transduction pathways. Depending on their levels, ROS may favor cell proliferation, neoplastic transformation, or cell death. The effects of ROS in cell turnover have been compared to a rheostat [26]: a moderate increase in ROS stimulates healthy resting cells to proliferate, and a further increase will favor tumor transformation. Excessive ROS production will lead to DNA damage and apoptotic cell death.

In agreement with observations made in isolated mitochondria, p13 was found to increase ROS production in several cell models (e.g. HeLa cells, primary $\mathrm{T}$ cells, and Jurkat T-ALL cells) [25]. These findings suggest that, in the context of the viral life cycle, p13 might contribute to an expansion of the pool of infected $\mathrm{T}$ cells, but trigger apoptosis of infected cells that acquire a transformed phenotype. In line with this model, the expression of p13 has been shown to lead to the activation of primary $\mathrm{T}$ cells and increased apoptotic death of Jurkat T-ALL cells [25].

\section{Role of $p 13$ in calcium signaling}

p13 also influences the mitochondrial uptake of calcium ions. In response to physiological stimuli (nutrients, hormones, neurotransmitters), $\mathrm{Ca}^{2+}$ rapidly accumulates in the mitochondrial matrix, where it stimulates enzymes of oxidative metabolism to produce ATP [27]. In contrast, mitochondrial calcium overload triggers sustained PTP opening, which leads to cell death [28].

Experiments carried out using $\mathrm{Ca}^{2+}$-sensitive aequorin probes localized to different cellular compartments (mitochondria, ER, or cytosol) showed that p13 inhibits the influx of $\mathrm{Ca}^{2+}$ into mitochondria, resulting in a local increase in the cytosolic concentration of $\mathrm{Ca}^{2+}$ (Fig. 3) [29]. This effect is interesting in light of the crucial role of $\mathrm{Ca}^{2+}$ signaling in the activation and survival of $\mathrm{T}$ cells, the primary targets of HTLV-1 infection. It would be interesting to determine if $\mathrm{p} 13$ functionally interacts with p12, a small, hydrophobic orf-I protein that localizes in the ER, binds calreticulin, and increases cytosolic $\mathrm{Ca}^{2+}$ levels, resulting in the activation of nuclear factor of activated $\mathrm{T}$ cells (NFAT) $[15,30]$. 


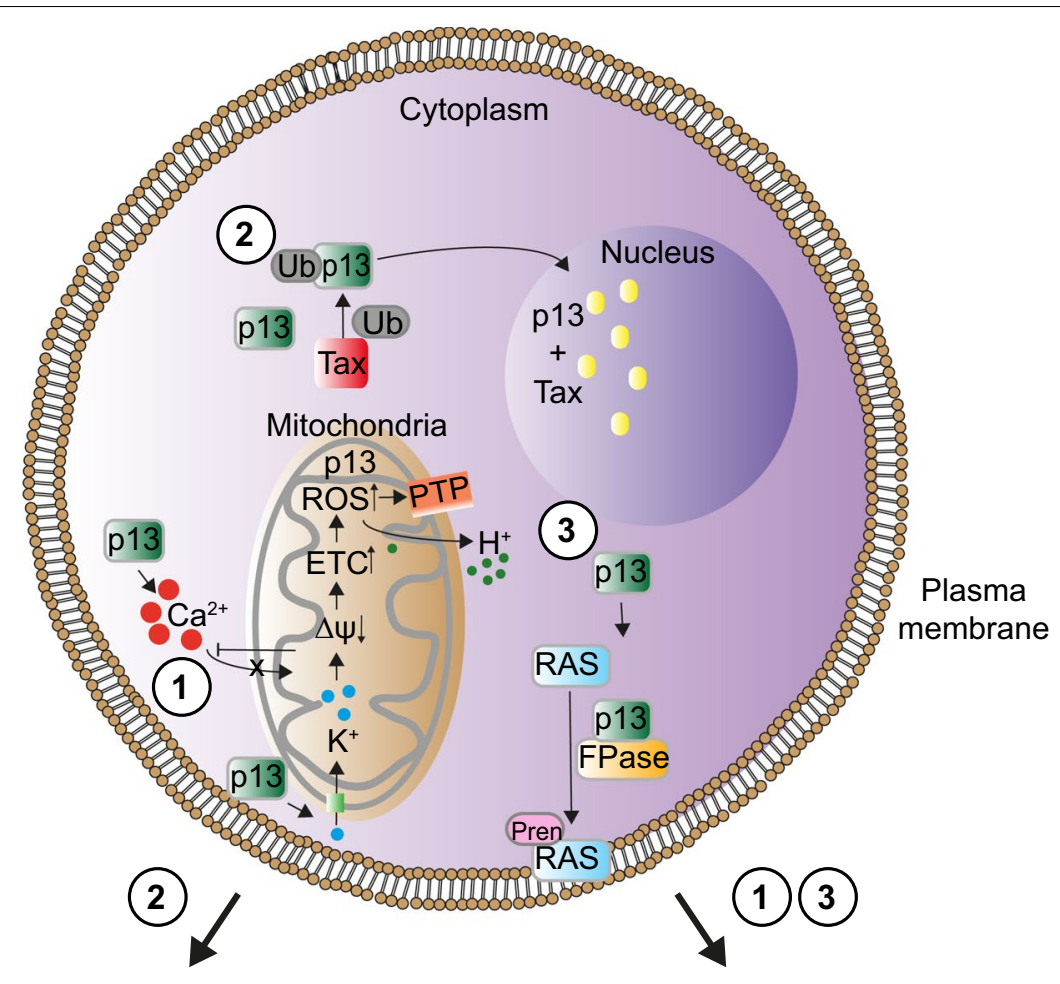

\section{Down-regulation of viral expression}

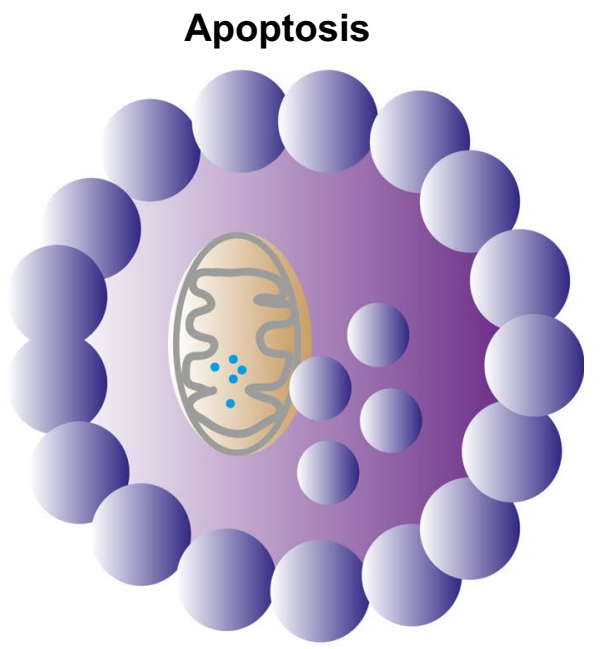

Fig. 3 Overview of p13 biology. (1) p13 accumulates in the inner mitochondrial membrane. It induces an inward $\mathrm{K}^{+}$current which leads to decreased mitochondrial membrane potential $(\Delta \psi)$ and a compensatory increase in the activity of the ETC, resulting in increased ROS production, and opening of the PTP, leading to mitochondrial swelling and apoptosis. p13 also interferes with the uptake of $\mathrm{Ca}^{2+}$ by mitochondria, which leads to an accumulation of cytosolic calcium. (2) When p13 is co-expressed with Tax, p13 becomes ubiquitinated and more stable. The protein partially accumulates in the nucleus, where p13 and Tax co-localize in nuclear speckles. (3) Interaction of p13 with farnesyl pyrophosphate synthase (FPase) interferes with Ras prenylation and targeting to the plasma membrane

\section{Effects of 13 on cell signaling pathways}

Lefebvre et al. showed that p13 negatively influences the Ras signal transduction pathway by binding to farnesyl pyrophosphate synthase (FPase), a key enzyme in the synthesis of substrates necessary for the prenylation of Ras and its association with the plasma membrane (Fig. 2) [31]. This property may contribute to the ability of p13 to suppress the transformation of rat embryo fibroblasts driven by the combination of Ras and Myc [32]. p13 also inhibits the proliferation 
of Jurkat cells when grown at high culture density and favors cell death under glucose deprivation or upon treatment with $\mathrm{C} 2$-ceramide, an inducer of the intrinsic pathway of apoptosis [33].

The C-terminal portion of p13 (and p30) is rich in prolines, including those arranged in a sequence resembling $\mathrm{P}-\mathrm{x}-\mathrm{x}-\mathrm{P}$ (PPII) helical motifs that interact with SH3 domain-containing proteins such as the Src family kinases (SFKs). Using in vitro binding and kinase assays, Tibaldi et al. confirmed that p13 is able to associate with SFKs Src, Fyn, Fgr, and Lyn [34]. This interaction led to increased kinase activity, but the effect was blocked in the presence of a 13 peptide spanning residues 61-87. Further analysis of the p13-Lyn interaction provided evidence that $\mathrm{p} 13$ is able to direct Lyn to mitochondria, resulting in an accumulation of p13Lyn complexes in the intermembrane space instead of the inner membrane location observed for p13 alone. This change in p13's submitochondrial localization was accompanied by an attenuation in the p13-mediated loss of mitochondrial membrane potential [34]. These p13-SFK interactions share some similarities with properties described for HIV-1 Nef, whose interactions with the Hck, Lyn, and Src SFKs play a positive role in viral replication $[35,36]$.

The role of autophagy in recycling damaged or unneeded organelles has gained increased attention in recent years. Mitophagy is a mitochondria-specific subtype of this recycling mechanism that can be induced by different factors, including viral infection [37]. Mitophagy induced by Hepatitis viruses $B$ and $C$ has been suggested to reduce apoptosis and increase viral persistence $[38,39]$. Coxsackie B virus (CVB) uses the mitophagy process for its viral spread [40], and classical swine fever virus (CSFV) induces mitophagy to inhibit apoptosis [41]. The effects of p13 on mitochondrial depolarization, swelling, and general homeostasis might therefore trigger the mitophagy process. The role of $\mathrm{p} 13$ in regulating essential parts of the mitophagy pathway would be an interesting point of investigation for future studies.

\section{Impact of 13 on Tax function}

Studies on the interplay between p13 and Tax [23] indicated that p13 binds directly to Tax and interferes with its ability to associate with $\mathrm{CBP} / \mathrm{p} 300$, an interaction that is needed for Tax-mediated activation of transcription from the long terminal repeat (LTR) promoter. Together with its late expression kinetics, this property supports a role for p13 as an 'off' switch that favors entrance into a latent state of infection, which is likely important for virus persistence in the host [23].

\section{p13-like proteins in other deltaretroviruses}

HTLV-1 is classified in the deltaretrovirus genus, together with the related HTLV-2, 3, and 4 orthologues that infect nonhuman primates as simian $\mathrm{T}$ cell leukemia virus (STLVs); and bovine leukemia virus (BLV). Due to their shared molecular features, the human and simian viruses are collectively referred to as primate T-lymphotropic viruses (PTLVs).

HTLV-2 circulates as 2 major subtypes, A and B, and is present mainly in South American indigenous populations, western and central Africa, and among intravenous drug users [42]. The pathogenicity of HTLV-2 is not clearly defined [43], but infection with this virus appears to significantly increase all-cause mortality [44]. The HTLV-2 $x$-II orf codes for a protein named p28 that, despite limited sequence identity, shares several functional properties with HTLV-1 p30 [45]. Transfection assays carried out using a plasmid coding for an HTLV-2A x-II orf carrying an epitope tag at the $3^{\prime}$ end did not reveal expression of any proteins smaller than p28, suggesting that HTLV-2A does not code for a p13 homologue [46]. Analogous experiments have not been carried out for HTLV-2B.

HTLV-3 [47, 48] and HTLV-4 [49] were identified in individuals from Cameroonian rainforests and are of unknown pathogenicity [50]. As part of a detailed analysis of the coding potential of HTLV-4 isolate 1863LE, Switzer et al. identified an open reading frame (orf- $I V)$ that codes for a 68 -amino acid protein with $75 \%$ similarity to portions of p13 located after the MTS and transmembrane motif [49]. Interestingly, alignment of this predicted protein with p13, HTLV-2 p28, and related orfs in STLV-2 (orf-II) and HTLV-3 (orf-III) revealed a highly conserved stretch of amino acids corresponding to p13 residues 59-84 in all of the sequences [49]. Afonso et al. [51] recently described a divergent STLV-1 isolate that contains mutations that affect splicing and/or codons that disrupt both the $\mathrm{x}$-II and $\mathrm{x}$-I orfs. An extension of this analysis to other STLVs and HTLVs showed disruption of either or both of these orfs in several other PTLVs [51].

BLV is a widespread pathogen that infects cattle, water buffalo, and zebus, and causes B-lymphocytosis, leukemia, and lymphoma. Accessory proteins coded by BLV include G4, a 105-amino acid protein that is targeted to mitochondria, and to a lesser extent, to the nucleus [31]. p13 and G4 share very little sequence identity (26.4\%, unpublished data), and its mitochondrial targeting depends on both an amino-proximal hydrophobic alpha-helix and a more carboxy-terminal arginine-rich amphipathic alpha helix [31]. In contrast to the tumor suppressor-like property observed for p13, BLV G4 
cooperates with $\mathrm{H}$-Ras in a rat embryo fibroblast transformation model, and is needed for BLV-driven tumor development in a sheep model [52].

\section{p13 and the host immune response}

Several studies over the past decade have revealed important roles for mitochondria in immune responses [53]. Many viruses encode mitochondrial proteins that are important for viral spread and persistence [54-56], and several viruses target MAVS (mitochondrial antiviralsignaling protein), a cellular protein localized in the outer mitochondrial membrane, mitochondrial-associated membranes, and peroxisomes that plays a critical role in innate immune response against RNA viruses [57-59]. The ability of HTLV-1 to infect monocytes suggests that the virus might impinge on the host's innate immune responses [60-63].

One way that p13 might impact the host's immune response could be through its regulation of cell death. Cell death mechanisms induced through mitochondrial pathways are known to enable the release of circular mitochondrial DNA (mtDNA) from mitochondria to the cytosol [64]. The presence of mtDNA in the cytosol can further trigger inflammatory responses in the host through the cyclic GMP-AMP synthase (cGAS)-stimulator of interferon genes (STING) signaling pathway $[65,66]$. The possible impact of $\mathrm{p} 13$ on anti-viral innate immunity is currently under investigation in our laboratories. The size and shape of mitochondria are controlled by the balance between mitochondrial fusion and fission [67]. This dynamic is intimately connected to the pattern of differentiation and activation of cells involved in immune responses. Naive effector T cells have small, round mitochondria, and their activation to effector $\mathrm{T}$ cells increases mitochondrial fission [68]. Mitochondrial fusion is prevalent in memory $\mathrm{T}$ cells, and results in elongated, tubular mitochondria and higher mitochondrial mass [68]. In their initial description of alternatively spliced HTLV-1 mRNAs, Berneman et al. showed that the p13 mRNA was expressed in 6 out of 10 ATLL samples, but was not detected in 3 PBMC samples from healthy HTLV-1 carriers [69]. It would be interesting to investigate if p13 might be involved in the selection of memory T-cells, which may represent the cell of origin of ATL [70].

\section{In vivo studies of $p 13$}

In vivo studies performed in rabbits using the molecular clone ACH.1 or a derivative containing a mutation that abolished expression of both orf-II proteins (ACH.30/13.1) showed that animals inoculated with ACH.30/13.1 had lower proviral loads than animals inoculated with ACH.1. These results suggest that p13 and/or p30 might be essential for the maintenance of viral loads in vivo [71]. The importance of p13 in vivo was confirmed in a more recent study of a molecular clone lacking the p13 initiation codon but still able to produce p30 [72]. However, in both studies the p13 knock-out was obtained by substituting its ATG start codon with GAT, which also disrupts the HBZ orf coded on the antisense strand [73]. New studies that do not interrupt the HBZ orf are thus needed to verify the importance of p13 alone.

\section{Sequence variation of $\mathrm{p} 13$}

While the HTLV-1 genome structure and sequence are in general highly conserved, sequence variations in HTLV-1 LTR segments are used to classify HTLV-1 isolates into 7 molecular subtypes with characteristic geographic distributions: subtypes A (further divided into 5 subgroups), B, C, D, E, F, and G [9]. Several studies have been directed at identifying subtypes and variants that might correlate with the clinical characteristics of infected patients. A study by Nozuma et al. comparing isolates from asymptomatic carriers and HAM/TSP patients confirmed the virus's high degree of sequence conservation but also provided evidence for an association between HAM/ TSP and infection with the "transcontinental" HTLV-1A subgroup [14]. Interestingly, this subgroup also showed a significantly higher frequency of mutations, mainly single nucleotide substitutions [14]. Kuramitsu investigated viral parameters associated with indeterminate HTLV-1 western blot (WB) results, defined as a lack of antibody reactivity against gp46Env and/or Gag proteins p53, p24 and $\mathrm{p} 19$, and observed that WB-indeterminate patients had a higher frequency of viral sequence variations compared to patients with positive WB results [13]. Even more notable was the median proviral load, which was almost 100-fold lower in this group. Kuramitsu et al. suggest that the low proviral load might be due to mutations in the provirus that reduce viral replication and limit dissemination within the host [13].

Using the datasets from these studies, we analyzed the p13 sequence in isolates from patients with different clinical characteristics. As a reference sequence, we used the p13 sequence coded by viral isolate ATK (accession number J02029.1), which was derived from a Japanese ATL patient and is considered to be a prototype for subtype A viruses [11]. A search using the Basic Local Alignment Search Tool (BLAST; https://blast.ncbi.nlm.nih.gov/Blast .cgi) yielded 69 hits obtained from published sequences $[12-14,74]$. Although the majority $(78 \%)$ of the sequences shared 100\% identity with p13 coded by ATK (not shown), 17 isolates showed amino acid variations (Fig. 4). Interestingly, two of these sequences have stop codons resulting in the early termination of p13 (isolates K1015 and 


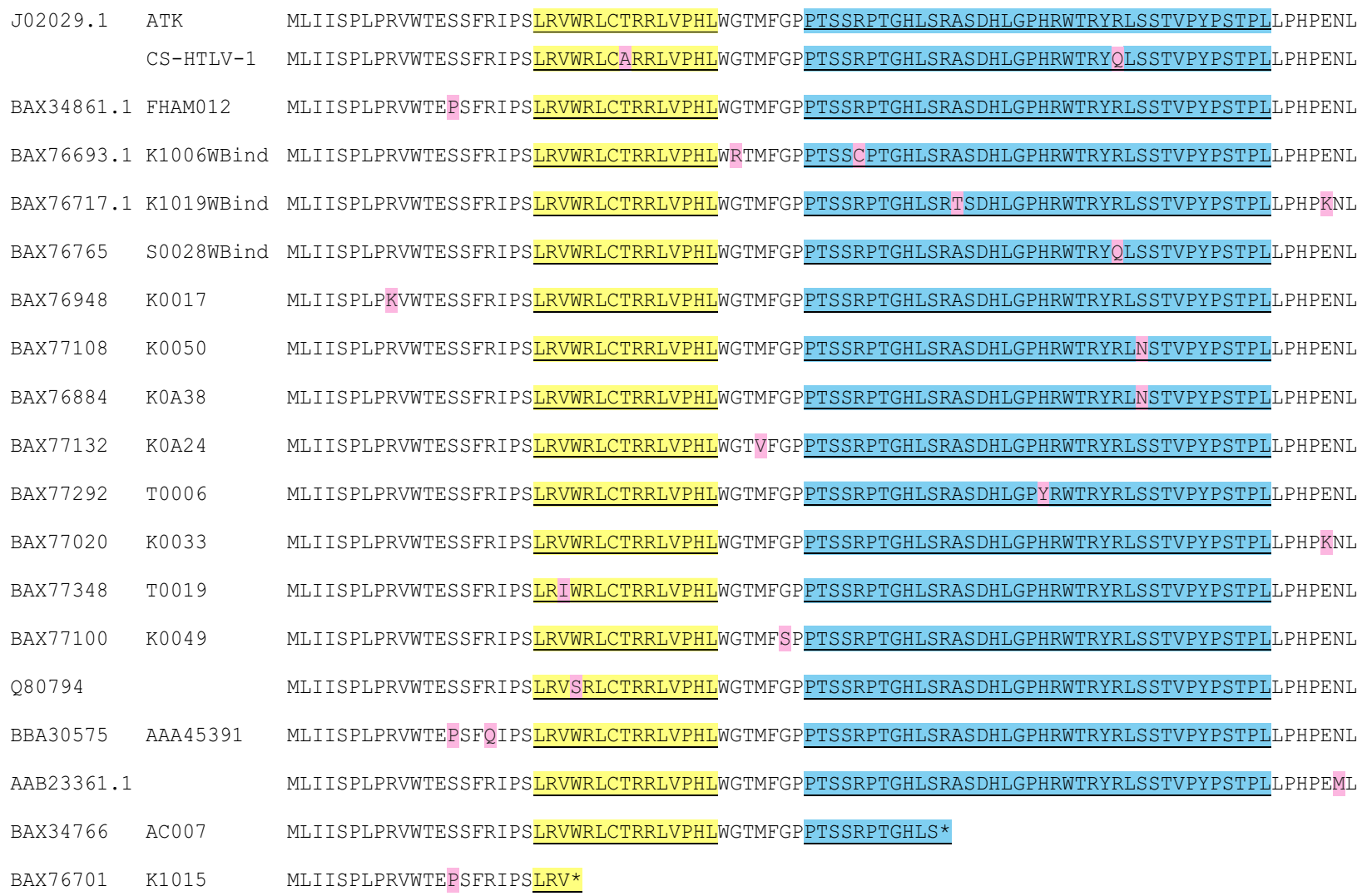

Fig. 4 HTLV-1A p13 sequence alignments. GenBank accession numbers and isolate names are indicated in the left column. Isolate CS-HTLV [75] is not deposited in GenBank. Its p13 sequence was determined by Sanger sequencing in our laboratory. Sequence variations are highlighted in pink. The mitochondrial targeting signal (MTS) and the putative nuclear localization sequence (NLS) are highlighted in yellow and blue, respectively. The multi-alignment was performed with the Mega7 program using default parameters

AC0007, Fig. 4). K1015, an isolate from the WB-indeterminate group [13], has an early termination codon at position 24 that results in the loss of all the domains essential for p13's intracellular targeting and functional properties. This isolate also has stop codons in Pol and Env (not shown). Isolate AC0007, from an asymptomatic carrier [14], has a stop codon at position 54 and thus retains the MTS and transmembrane sequence, but lacks most of the putative nuclear localization sequence (NLS) of p13 (Fig. 4). Two other isolates have a single amino acid difference in the MTS domain. None of the sequences in the BLAST search matched with p13 coded by the CS-clone of HTLV-1, which was derived from a North American ATL patient [75] and carries a single amino acid difference in the MTS and one in the putative NLS. All of the samples with variations in the MTS were from HAM/TSP or ATL patients who had positive WB results. None of the variations in the MTS involve the four arginines identified as critical for p13 function [20].
Amino acid variations within p13's putative NLS were identified in six of the sequences: three in the WBindeterminate group, and three in the WB-positive group (Fig. 4) [13]. Two of the latter sequences carried an asparagine in place of serine at position $70(\mathrm{~S} 70 \mathrm{~N})$. Although Kuramitsu et al. concluded that the samples from the group with indeterminate WB results had the highest frequency of sequence variations [13], the majority of isolates with p13 variations were from patients with either an HTLV-1 associated disease or detectable antigens in WB analysis. Further studies are needed to determine if the variations observed in the patients with positive WB results or disease actually influence the function of p13. It is notable that the two isolates coding for truncated p13 proteins were obtained from asymptomatic carriers who did not have positive WB results, suggesting a possible link between p13 and disease development. 
(See figure on next page.)

Fig. 5 Alignments of p13 coded by HTLV-1A prototype ATK and 37 HTLV-1C isolates. GenBank accession numbers and/or isolate names are indicated in the left column. Patient isolates 9-31 are described in [82]. Isolates from Australia are indicated in bold. Isolates NCP201, VAN-EM5, and MEL5 were obtained from New Caledonia, Vanuatu [84], and the Solomon Islands [85], respectively. Sequence variations are highlighted in pink. The mitochondrial targeting signal (MTS) and the putative nuclear localization sequence (NLS) are highlighted in yellow and blue, respectively. Arrows at the bottom of the figure indicate an 8-residue 'signature' present in all Australian isolates (see text). The multi-alignment was performed with the Mega7 program using default parameters

\section{Sequence variations in $\mathrm{p} 13$ coded by HTLV-1 subtypes $A$ and $C$}

HTLV-1 C, present mainly in Australia and Melanesia, has recently gained attention due to it high prevalence (in up to $40 \%$ of adults) in indigenous populations living in remote regions of central Australia [76]. Unlike subtype A, subtype $C$ seems to be more frequently associated with inflammatory disease in the lungs, causing bronchiectasis or bronchitis with high morbidity [77, 78]. However, cases of infectious dermatitis and ATL have also been reported for HTLV-1C [79, 80]. The reasons for the different pathogenic properties of the two subtypes are still unclear.

Pairwise sequence comparisons at the nucleotide and amino acid levels between HTLV-1A and HTLV-1C show that the structural genes, gag, pol, pro, and env are more highly conserved compared to the $\mathrm{pX}$ region, suggesting that the $\mathrm{X}$-region proteins might contribute to the different clinical manifestations observed in infected individuals [81]. To gain additional insight into the role of p13 in HTLV-1 pathogenesis, we extended our analysis to the p13 proteins coded by 37 subtype $\mathrm{C}$ sequences, 34 of which were isolated from Australian patients (Fig. 5) [82-85]. Sequence information for 4 amino-terminal amino acids was not available for 8 GenBank entries (prefix KC in Fig. 5). Since these amino acids were invariant in the other sequences, we chose to compare amino acids 5-87 for the full set of sequences. The analysis revealed 89-95\% identity between ATK and the 37 HTLV-1C sequences, 90-100\% identity among all HTLV-1C sequences, and $98-100 \%$ identity among the 34 Australian sequences, with 31 out of 34 showing 100\% identity. Interestingly, all 34 Australian sequences share an 8-amino acid 'signature' that distinguishes them from ATK. The first three residues of this signature are present in the entire set of HTLV-1C sequences (Fig. 5).

Overall, this analysis confirms similar amino acid sequences and conservation of the principal domains described for HTLV-1A p13, including the MTS, the transmembrane region, the putative NLS region and the
P-x-x-P SH3 binding motif. It is notable that the MTS is perfectly matched among all of the sequences. Interestingly, three of the 'Australian signature' residues are located in the putative NLS region, and the last signature residue is a carboxy-terminal proline, which would add a third potential $\mathrm{P}-\mathrm{x}-\mathrm{x}-\mathrm{P}$ motif.

Whether these changes affect the function of p13 and contribute to the distinct pathogenic properties of the HTLV-1C clade found in indigenous Australian patients [83] should be a point of investigation in future studies.

\section{Conclusions}

Many studies have shed light on the expression, intracellular trafficking, and function of p13, but the function of this protein in the context of the complete viral genome remains elusive. Given the importance of mitochondria in anti-viral innate immunity, it will also be important to discover if, by targeting mitochondria, p13 may hamper the host cell's anti-viral responses. Notably, several other human tumor viruses also code for mitochondrial proteins, with diverse effects on mitochondrial function and the viral life cycle [56].

In vivo study of a molecular clone of HTLV-1 without changing the start codon of HBZ is essential for determining the true importance of p13 in HTLV-1 infection. Galli et al. showed the possibility of utilizing humanized mice for studying the 12 protein of HTLV-1 in the whole virus using infected primary $\mathrm{CD}^{+}{ }^{+}$cells [86]. This model may be useful for better assessing the pathogenic potential of p13 and investigating the intracellular localization of the protein in different tissues in infected animals in vivo. The identification of new roles for mitochondria opens up new questions about the role of p13 in both the pathogenesis of HTLV-1 and in immune responses. HTLV-1C involves up to half of some communities in endemic foci in Australia. The remarkable concentration of this virus demands that the functional role of p13 be better understood, and presents an opportunity to investigate the effects of the different sequence variations in the functional domains of $\mathrm{p} 13$. 


\begin{tabular}{|c|c|c|}
\hline J02029.1 & ATK & MLI ISPLPRVWTESSFRIPSLRVWRLCTRRLVPHLWGTMFGPPTSSRPTGHLSRASDHLGPHRWTRYRLSSTVPYPSTPLLPHPENL \\
\hline JX891479 & AUS-NR & MLI ISPLPRVWTKSSLRISSLRVWRLCTRRLVPHLWGTMLGPPTSSRPTGHLPRTSDHLGPHRWTRYRLSSTIPYPSTPLLPHPENP \\
\hline KF242505 & AUS-DF & MLI ISPLPRVWTKSSLRISSLRVWRLCTRRLVPHLWGTMLGPPTSSRPTGHLPRTSDHLGPHRWTRYRLSSTIPYPSTPLLPHPENP \\
\hline KF242506 & AUS-CS & MLI ISPLPRVWTKSSLRISSLRVWRLCTRRLVPHLWGTMLGPPTSSRPTGHLPRTSDHLGPHRWTRYRLSSTIPYPSTPLLPHPENP \\
\hline JX891478 & AUS-GM & MLIISPLPRVWTKSSLRISSLRVWRLCTRRLVPHLWGTMLGPPTSSRPTGHLPRTSDHLGPHRWTRYRLSSTIPYPSTPLLPHPENP \\
\hline KC786941 & AUS-GN & ---?SPLPRVWTKSSLRISSLRVWRLCTRRLVPHLWGTMLGPPTSSRPTGHLPRTSDHLGPHRWTRYRLSSTIPYPSTPLLPHPENP \\
\hline KC786942 & AUS-NA & ---?SPLPRVWTKSSLRISSLRVWRLCTRRLVPHLWGTMLGPPTSSRPTGHLPRTSDHLGPHRWTRYRLSSTIPYPSTPLLPHPENP \\
\hline KC786943 & AUS-SQ & ---?SPLPRVWTKSSLRISSLRVWRLCTRRLVPHLWGTMLGPPTSSRPTGHLPRTSDHLGPHRWTRYRLSSTIPYPSTPLLPHPENP \\
\hline KC786944 & AUS-MI & PPTSSRPTGHLPRTSDHLGPHRWTRYRLSSTIPYPSTPLLPHPENP \\
\hline KC786947 & AUS-AN & PTSSRPTGHLPRTSDHLGPHRWTRYRLSSTIPYPSTPLLPHPENP \\
\hline KC786949 & AUS-DM & PTSSRPTGHLPRTSDHLGPHRWTRYRLSSTIPYPSTPLLPHPENP \\
\hline KC786952 & AUS-RI & ---?SPLPRVWTKSSLRISSLRVWRLCTRRLVPHLWGTMLGPPTSSRPTGHLPRTSDHLGPHRWTRYRLSSTIPYPSTPLLPHPENP \\
\hline KC786958 & AUS-LT & ---?SPLPRVWTKSSLRISSLRVWRLCTRRLVPHLWGTMLGPPTSSRPTGHLPRTSDHLGPHRWTRYRLSSTIPYPSTPLLPHPENP \\
\hline KX905203 & NCP201 & MLI ISPLPRVWTKSSLRISSLRVWRLCTRRLVPHLWGTMFGPLTSSRPTGHLSGASDHLGPHRWTRYRLSSTIPYPSAPLLPHPENL \\
\hline KX905202.1 & VAN-EM5 & MLI ISPLPRVWTKSSLRISSLRVWRLCTRRLVPHLWGTMFGPPTSSRPTGHLSGASDHLGPHRWTRYRLSSTIPYPSAPLLPHPENL \\
\hline L02534 & MEL5 & MLI ISPLPRVWTKKSSLRISSLRVWRLCTRRLVPHLWGTMFGPPTSSRPTGHLSRASDHLGPHRWTRYRLSSTVPYPSAPLLPHPENL \\
\hline patient 9 & & MLI ISPLPRVWTKSSLRISSLRVWRLCTRRLVPHLWGTMLGPPTSSRPTGHLPRTSDHLGPHRWTRYRLSSTIPYPSTPLLPHPENP \\
\hline patient 10 & & TSSRPTGHLPRTSDHLGPHRWTRYRLSSTIPYPSTPLLPHPENP \\
\hline patient 11 & & PTSSRPTGHLPRTSDHLGPHRWTRYRLSSTIPYPSTPLLPHPENP \\
\hline patient 12 & & PTSSRPTGHLPRTSDHLGPHRWTRYRLSSTIPYPSTPLLPHPENP \\
\hline patient 13 & & MLI ISPLPRVWTKSSLRISSLRVWRLCTRRLVPHLWGTMLGPPTSSRPTGHLPRTSDHLGPHRWTRYRLSSTIPYPSTPLLPHPENP \\
\hline patient 14 & & MLI ISPLPRVWTKSSLRISSLRVWRLCTRRLVPHLWGTMLGPPTSSRPTGHLPRTSDHLGPHR?TRYRLS?TIPYPSTPLLPHPENP \\
\hline patient 15 & & MLIISPLPRVWTKSSLRISSLRVWRLCTRRLVPHLWGTMLGPPTSSRPTGHLPRTSDHLGPHRWTRYRLSSTIPYPSTPLLPHPENP \\
\hline patient 16 & & MLI ISP? PRVGTKSSLRISSLRVWRLCTRRLVPHLWGTMLGPPTSSRPTGHLPRT SDHLGPHRWTRYRLSSTIPYPSTPLLPHPENP \\
\hline patient 17 & & MLI ISPLPRVWTKSSLRISSLRVWRLCTRRLVPHLWGTMLGPPTSSRPTGHLPRTSDHLGPHRWTRYRLSSTIPYPSTPLLPHPENP \\
\hline patient 18 & & MLI ISPLPRVWTKSSLRISSLRVWRLCTRRLVPHLWGTMLGPPTSSRPTGHLPRTSDHLGPHRWTRYRLSSTIPYPSTPLLPHPENP \\
\hline patient 19 & & MLI ISPLPRVWTKSSLRISSLRVWRLCTRRLVPHLWGTMLGPPTSSRPTGHLPRTSDHLGPHRWTRYRLSSTIPYPSTPLLPHPENP \\
\hline patient 20 & & PTSSRPTGHLPRTSDHLGPHRWTRYRLSSTIPYPSTPLLPHPENP \\
\hline patient 21 & & MLI ISPLPRVWTKSSLRISSLRVWRLCTRRLVPHLWGTMLGPPTSSRPTGHLPRTSDHLGPHRWTRYRLSSTIPYPSTPLLPHPENP \\
\hline patient 22 & & MLI ISPLPRVWTKSSLRISSLRVWRLCTRRLVPHLWGTMLGPPTSSRPTGHLPRTSDHLGPHRWTRYRLSSTIPYPSTPLLPHPENP \\
\hline patient 23 & & MLIISPLPRVWTKSSLRISSLRVWRLCTRRLVPHLWGTMLGPSTSSRPTGHLPRTSDHLGPHRWTRYRLSSTIPYPSTPLLPHPENP \\
\hline patient 24 & & MLI ISPLPRVWTKSSLRIS?LRVWRLCTRRLVPHLWGTMLGPPTSSRPTGHLPRTSDHLGPHRWTRYRLSSTIPYPSTPLLPHPENP \\
\hline patient 25 & & MLIISPLPRVWTKSSLRISSLRVWRLCTRRLVPHLWGTMLGP \\
\hline patient 26 & & MLIISPLPRVWTKSSLRISSLRVWRLCTRRLVPHLWGTMLG \\
\hline patient 27 & & TSSRPTGHLPRTSDHLGPHRWTRYRLSSTIPYPSTPLLPHPENP \\
\hline patient 29 & & TSSRPTGHLPRTSDHLGPHRWTRYRLSSTIPYPSTPLLPHPENP \\
\hline patient 30 & & MLIISPLPRVWTKSSLRISSLRVWRLCTRRLVPHLWGTMLGPPTSSRPTGHLPRTSDHLGPHRWTRYRLSSTIPYPSTPLLPHPENP \\
\hline patient 31 & & MLIISPLPRVWTKSSLRISSLRVWRLCTRRLVPHLWGTMLGPPTSSRPTGHLPRTSDHLGPHRWTRYRLSSTIPYPSTPLLPHPENP \\
\hline
\end{tabular}




\section{Abbreviations}

ATL: Adult T cell leukemia; BLAST: Basic local alignment search tool; CSFV: Classical swine fever virus; CVB: Coxsackie B virus; cGAS: Cyclic GMP-AMP synthase; ER: Endoplasmic reticulum; ETC: Electron transport chain; FPase: Farnesyl pyrophosphate synthase; GFP: Green fluorescent protein; HA: Hemagglutinin; HBZ: HTLV-1 bZIP factor; HIV: Human immunodeficiency virus; HSP: Heat shock protein; HTLV: Human T cell leukemia virus; HAM/TSP: HTLV-1 associated myelopathy/tropical spastic paraparesis; LTR: Long terminal repeat; MAVS: Mitochondrial antiviral-signaling protein; mtDNA: mitochondrial DNA; MTS: Mitochondrial targeting signal; NLS: Nuclear localization sequence; NFAT: nuclear factor of activated T cells; orf: Open reading frame; PTLV: Primate T cell leukemia virus; PTP: Permeability transition pore; ROS: Reactive oxygen species; SFK: Src family kinase; SH3: Src homology 3; STING: Stimulator of interferon genes; STLV: Simian T cell leukemia virus; T-ALL: T cell acute lymphoblastic leukemia; WB: Western blot.

\section{Acknowledgements}

We thank D. Ahern for editorial support.

\section{Authors' contributions}

MO, MSB, SS, RM, DMD, GF, and VC wrote the manuscript.; MO aligned sequences and prepared figures; VC provided data shown in Fig. 3, GK, DY, and DFJP provided HTLV-1C sequence information; MSB performed the experiments in Fig. 3; DMD and SS aligned HTLV-1C sequences; All authors read and approved the final manuscript.

\section{Funding}

This work was supported by the Intramural Research Program of the National Institutes of Health, National Cancer Institute, Center for Cancer Research, and federal funds from the National Cancer Institute, National Institutes of Health, a grant from the Italian Association for Cancer Research (AIRC) IG \# 17794 to VC, institutional funds from the University of Padua (to VC and DMD), and the National Health and Medical Research Council of Australia (APP1129320 and APP1052979 to DFJP).

\section{Availability of data and materials}

All data generated or analyzed during this study are included in published articles cited in the review and/or are available in GenBank https://www.ncbi. nlm.nih.gov/genbank/.

\section{Ethics approval and consent to participate}

HTLV-1C p13 sequences from infected individuals were obtained from blood DNA obtained with informed consent in first language and written consent submitted in accordance with the National Health and Medical Research Council of Australia. This study was approved by the Central Australian Human Research Ethics Committee (CAHREC; Reference HREC-14-249).

\section{Consent for publication}

Not applicable.

\section{Competing interests}

The authors declare that they have no competing interests.

\section{Author details}

${ }^{1}$ Animal Models and Retroviral Vaccines Section, Vaccine Branch, Center for Cancer Research, National Cancer Institute, National Institutes of Health, Bethesda, MD, USA. ${ }^{2}$ Veneto Institute of Oncology IOV-IRCCS, Padua, Italy. ${ }^{3}$ Department of Microbiology and Immunology, The Peter Doherty Institute for Infection and Immunity, University of Melbourne, Parkville, VIC, Australia. ${ }^{4}$ Department of Biomedical Sciences, University of Padua, Padua, Italy. ${ }^{5}$ Department of Surgery, Oncology, and Gastroenterology, University of Padua, Padua, Italy. ${ }^{6}$ Present Address: Division of Microbiology and Immunology, Yerkes National Primate Research Center, Emory University, Atlanta, GA, USA.

Received: 23 January 2020 Accepted: 10 April 2020

Published online: 12 May 2020

\section{References}

1. Poiesz BJ, et al. Detection and isolation of type $C$ retrovirus particles from fresh and cultured lymphocytes of a patient with cutaneous T-cell lymphoma. Proc Natl Acad Sci USA. 1980;77(12):7415-9.

2. Nakao K, Ohba N. Clinical features of HTLV-I associated uveitis. Br J Ophthalmol. 1993;77(5):274-9.

3. Nishioka K, et al. Chronic inflammatory arthropathy associated with HTLVI. Lancet. 1989;1(8635):441.

4. LaGrenade $L$, et al. Infective dermatitis of Jamaican children: a marker for HTLV-I infection. Lancet. 1990;336(8727):1345-7.

5. Ohshima K, et al. Human T-cell leukemia virus type I associated lymphadenitis. Cancer. 1992;69(1):239-48.

6. Eguchi K, et al. Primary Sjogren's syndrome with antibodies to HTLV-l: clinical and laboratory features. Ann Rheum Dis. 1992;51(6):769-76.

7. Gessain A, et al. Antibodies to human T-lymphotropic virus type-l in patients with tropical spastic paraparesis. Lancet. 1985;2(8452):407-10.

8. Osame M, et al. HTLV-I associated myelopathy, a new clinical entity. Lancet. 1986;1(8488):1031-2.

9. Gessain A, Cassar O. Epidemiological aspects and world distribution of HTLV-1 infection. Front Microbiol. 2012;3:388.

10. Giam CZ, Semmes OJ. HTLV-1 infection and adult T-cell leukemia/ Iymphoma-a tale of two proteins: Tax and HBZ. Viruses. 2016;8(6):161.

11. Seiki M, et al. Human adult T-cell leukemia virus: complete nucleotide sequence of the provirus genome integrated in leukemia cell DNA. Proc Natl Acad Sci USA. 1983;80(12):3618-22.

12. Koralnik IJ, et al. Protein isoforms encoded by the $\mathrm{pX}$ region of human T-cell leukemia/lymphotropic virus type I. Proc Natl Acad Sci USA. 1992;89(18):8813-7.

13. Kuramitsu $M$, et al. Proviral features of human $T$ cell leukemia virus type 1 in carriers with indeterminate western blot analysis results. J Clin Microbiol. 2017:55(9):2838-49.

14. Nozuma S, et al. Effects of host restriction factors and the HTLV-1 subtype on susceptibility to HTLV-1-associated myelopathy/tropical spastic paraparesis. Retrovirology. 2017;14(1):26.

15. Koralnik IJ, Fullen J, Franchini G. The p12I, p13II, and p30II proteins encoded by human T-cell leukemia/lymphotropic virus type I open reading frames I and II are localized in three different cellular compartments. J Virol. 1993;67(4):2360-6.

16. Ciminale V, et al. Complex splicing in the human T-cell leukemia virus (HTLV) family of retroviruses: novel mRNAs and proteins produced by HTLV type I. J Virol. 1992;66(3):1737-45.

17. Rende F, et al. Kinetics and intracellular compartmentalization of HTLV-1 gene expression: nuclear retention of HBZ mRNAs. Blood. 2011;117(18):4855-9.

18. Cavallari I, et al. Expression of alternatively spliced human T-Cell leukemia virus type 1 mRNAs is influenced by mitosis and by a novel cis-acting regulatory sequence. J Virol. 2016;90(3):1486-98.

19. Ciminale $V$, et al. Mitochondrial targeting of the p13ll protein coded by the $x$-II ORF of human T-cell leukemia/lymphotropic virus type I (HTLVI). Oncogene. 1999;18(31):4505-14.

20. D'Agostino DM, et al. Mitochondrial alterations induced by the p13/I protein of human T-cell leukemia virus type 1. Critical role of arginine residues. J Biol Chem. 2002;277(37):34424-33.

21. D'Agostino DM, et al. The human T-cell leukemia virus type 1 p13॥ protein: effects on mitochondrial function and cell growth. Cell Death Differ. 2005;12(Suppl 1):905-15.

22. Ghorbel S, et al. Human T-cell leukemia virus type I p30 nuclear/ nucleolar retention is mediated through interactions with RNA and a constituent of the $60 \mathrm{~S}$ ribosomal subunit. J Biol Chem. 2006;281(48):37150-8.

23. Andresen $V$, et al. Suppression of HTLV-1 replication by Tax-mediated rerouting of the p13 viral protein to nuclear speckles. Blood. 2011;118(6):1549-59.

24. Silic-Benussi $M$, et al. Modulation of mitochondrial $K(+)$ permeability and reactive oxygen species production by the p13 protein of human T-cell leukemia virus type 1. Biochim Biophys Acta. 2009;1787(7):947-54.

25. Silic-Benussi $M$, et al. Redox regulation of T-cell turnover by the p13 protein of human T-cell leukemia virus type 1: distinct effects in primary versus transformed cells. Blood. 2010;116(1):54-62. 
26. Trachootham D, Alexandre J, Huang P. Targeting cancer cells by ROSmediated mechanisms: a radical therapeutic approach? Nat Rev Drug Discov. 2009;8(7):579-91.

27. Tarasov Al, Griffiths EJ, Rutter GA. Regulation of ATP production by mitochondrial Ca(2+). Cell Calcium. 2012;52(1):28-35.

28. Parks RJ, Murphy E, Liu JC. Mitochondrial permeability transition pore and calcium handling. Methods Mol Biol. 2018;1782:187-96.

29. Biasiotto $R$, et al. The $p 13$ protein of human $T$ cell leukemia virus type 1 (HTLV-1) modulates mitochondrial membrane potential and calcium uptake. Biochim Biophys Acta. 2010;1797(6-7):945-51.

30. Ding W, et al. Human T-cell lymphotropic virus type 1 p12(l) expression increases cytoplasmic calcium to enhance the activation of nuclear factor of activated T cells. J Virol. 2002;76(20):10374-82.

31. Lefebvre L, et al. Oncoviral bovine leukemia virus $\mathrm{G} 4$ and human T-cell leukemia virus type 1 p13(II) accessory proteins interact with farnesyl pyrophosphate synthetase. J Virol. 2002;76(3):1400-14.

32. Silic-Benussi $M$, et al. Suppression of tumor growth and cell proliferation by p13II, a mitochondrial protein of human T cell leukemia virus type 1. Proc Natl Acad Sci USA. 2004;101(17):6629-34.

33. Hiraragi $\mathrm{H}$, et al. Human T-lymphotropic virus type 1 mitochondrionlocalizing protein p13ll sensitizes Jurkat T cells to Ras-mediated apoptosis. J Virol. 2005;79(15):9449-57.

34. Tibaldi $\mathrm{E}$, et al. Interaction between the $\mathrm{SH} 3$ domain of Src family kinases and the proline-rich motif of HTLV-1 p13: a novel mechanism underlying delivery of Src family kinases to mitochondria. Biochem J. 2011;439(3):505-16.

35. Saksela K, Cheng G, Baltimore D. Proline-rich (PxxP) motifs in HIV-1 Nef bind to $\mathrm{SH} 3$ domains of a subset of Src kinases and are required for the enhanced growth of Nef+ viruses but not for down-regulation of CD4. EMBO J. 1995;14(3):484-91.

36. Trible RP, Emert-Sedlak L, Smithgall TE. HIV-1 Nef selectively activates Src family kinases Hck, Lyn, and C-Src through direct SH3 domain interaction. J Biol Chem. 2006;281(37):27029-38.

37. Zhang $L$, Qin $Y$, Chen M. Viral strategies for triggering and manipulating mitophagy. Autophagy. 2018;14(10):1665-73.

38. Kim SJ, et al. Hepatitis B virus disrupts mitochondrial dynamics: induces fission and mitophagy to attenuate apoptosis. PLoS Pathog. 2013:9(12):e1003722

39. Kim SJ, et al. Hepatitis C virus triggers mitochondrial fission and attenuates apoptosis to promote viral persistence. Proc Natl Acad Sci USA 2014;111(17):6413-8.

40. Sin J, et al. Coxsackievirus B Escapes the infected cell in ejected mitophagosomes. J Virol. 2017;91(24):e01347-17.

41. Gou H, et al. CSFV induced mitochondrial fission and mitophagy to inhibit apoptosis. Oncotarget. 2017;8(24):39382-400.

42. Roucoux DF, Murphy EL. The epidemiology and disease outcomes of human T-lymphotropic virus type II. AIDS Rev. 2004;6(3):144-54.

43. Ciminale V, et al. HTLV-1 and HTLV-2: highly similar viruses with distinct oncogenic properties. Front Microbiol. 2014;5:398.

44. Biswas HH, et al. Increased all-cause and cancer mortality in HTLV-II infection. J Acquir Immune Defic Syndr. 2010;54(3):290-6.

45. Harrod R. Silencers of HTLV-1 and HTLV-2: the pX-encoded latencymaintenance factors. Retrovirology. 2019;16(1):25.

46. Ciminale $V$, et al. Expression and characterization of proteins produced by mRNAs spliced into the $X$ region of the human T-cell leukemia/lymphotropic virus type II. Virology. 1995;209(2):445-56.

47. Calattini $\mathrm{S}$, et al. Discovery of a new human T-cell lymphotropic virus (HTLV-3) in Central Africa. Retrovirology. 2005;2:30.

48. Wolfe ND, et al. Emergence of unique primate T-lymphotropic viruses among central African bushmeat hunters. Proc Natl Acad Sci USA. 2005;102(22):7994-9

49. Switzer WM, et al. Ancient, independent evolution and distinct molecular features of the novel human T-lymphotropic virus type 4. Retrovirology. 2009;6:9.

50. Mahieux R, Gessain A. HTLV-3/STLV-3 and HTLV-4 viruses: discovery, epidemiology, serology and molecular aspects. Viruses. 2011;3(7):1074-90

51. Afonso PV, et al. Absence of accessory genes in a divergent simian T-lymphotropic virus type 1 isolated from a bonnet macaque (Macaca radiata). PLoS Negl Trop Dis. 2019;13(7):e0007521.
52. Kerkhofs $P$, et al. In vitro and in vivo oncogenic potential of bovine leukemia virus G4 protein. J Virol. 1998;72(3):2554-9.

53. Mills EL, Kelly B, O'Neill LAJ. Mitochondria are the powerhouses of immunity. Nat Immunol. 2017;18(5):488-98.

54. Boya P, et al. Viral proteins targeting mitochondria: controlling cell death. Biochim Biophys Acta. 2004;1659(2-3):178-89.

55. D'Agostino DM, et al. Mitochondria as functional targets of proteins coded by human tumor viruses. Adv Cancer Res. 2005;94:87-142.

56. Cavallari l, et al. Mitochondrial proteins coded by human tumor viruses. Front Microbiol. 2018;9:81.

57. Hou F, et al. MAVS forms functional prion-like aggregates to activate and propagate antiviral innate immune response. Cell. 2011;146(3):448-61.

58. Koshiba T. Mitochondrial-mediated antiviral immunity. Biochimica Et Biophysica Acta Mol Cell Res. 2013;1833(1):225-32.

59. Vazquez C, Horner SM. MAVS coordination of antiviral innate immunity. J Virol. 2015;89(14):6974-7.

60. Makino $M$, et al. The role of human T-lymphotropic virus type 1 (HTLV1)-infected dendritic cells in the development of HTLV-1-associated myelopathy/tropical spastic paraparesis. J Virol. 1999;73(6):4575-81.

61. Enose-Akahata Y, et al. Retrovirally induced CTL degranulation mediated by IL-15 expression and infection of mononuclear phagocytes in patients with HTLV-I-associated neurologic disease. Blood. 2008:112(6):2400-10.

62. Koyanagi Y, et al. In vivo infection of human T-cell leukemia virus type I in non-T cells. Virology. 1993;196(1):25-33.

63. de Castro-Amarante MF, et al. Human T cell leukemia virus type 1 infection of the three monocyte subsets contributes to viral burden in humans. J Virol. 2015;90(5):2195-207.

64. Sinigaglia F, Richard J, Pink L. Human T lymphocyte clones specific for malaria (Plasmodium falciparum) antigens. EMBO J. 1985;4(13B):3819-22.

65. Riley JS, et al. Mitochondrial inner membrane permeabilisation enables mtDNA release during apoptosis. EMBO J. 2018;37(17):e99238.

66. West AP, Shadel GS. Mitochondrial DNA in innate immune responses and inflammatory pathology. Nat Rev Immunol. 2017;17(6):363-75.

67. Rambold AS, Pearce EL. Mitochondrial dynamics at the interface of immune cell metabolism and function. Trends Immunol. 2018;39(1):6-18.

68. Ron-Harel N, et al. Mitochondrial biogenesis and proteome remodeling promote one-carbon metabolism for T cell activation. Cell Metab. 2016:24(1):104-17.

69. Berneman ZN, et al. Expression of alternatively spliced human T-lymphotropic virus type I pX mRNA in infected cell lines and in primary uncultured cells from patients with adult T-cell leukemia/lymphoma and healthy carriers. Proc Natl Acad Sci USA. 1992;89(7):3005-9.

70. Nagai $Y$, et al. T memory stem cells are the hierarchical apex of adult T-cell leukemia. Blood. 2015;125(23):3527-35.

71. Bartoe JT, et al. Functional role of $\mathrm{pX}$ open reading frame II of human T-lymphotropic virus type 1 in maintenance of viral loads in vivo. J Virol. 2000;74(3):1094-100.

72. Hiraragi $\mathrm{H}$, et al. Human T-lymphotropic virus type 1 mitochondrionlocalizing protein p13(II) is required for viral infectivity in vivo. J Virol. 2006;80(7):3469-76.

73. Gaudray G, et al. The complementary strand of the human T-cell leukemia virus type 1 RNA genome encodes a bZIP transcription factor that down-regulates viral transcription. J Virol. 2002;76(24):12813-22.

74. Tsujimoto A, et al. Nucleotide sequence analysis of a provirus derived from HTLV-1-associated myelopathy (HAM). Mol Biol Med. 1988:5(1):29-42.

75. Derse D, et al. Virions released from cells transfected with a molecular clone of human T-cell leukemia virus type I give rise to primary and secondary infections of T cells. J Virol. 1995;69(3):1907-12.

76. Einsiedel $L J$, et al. The prevalence and clinical associations of HTLV-1 infection in a remote Indigenous community. Med J Aust. 2016;205(7):305-9.

77. Einsiedel $L$, et al. Bronchiectasis is associated with human T-lymphotropic virus 1 infection in an Indigenous Australian population. Clin Infect Dis. 2012:54(1):43-50. 
78. Einsiedel L, et al. Human T-Lymphotropic Virus type 1c subtype proviral loads, chronic lung disease and survival in a prospective cohort of Indigenous Australians. PLoS Negl Trop Dis. 2018;12(3):e0006281.

79. Einsiedel L, et al. Human T-lymphotropic virus type 1 infective dermatitis in central Australia. J Clin Virol. 2013;57(4):370-3.

80. Kirkland MA, Frasca J, Bastian I. Adult T-cell leukaemia lymphoma in an aborigine. Aust N Z J Med. 1991;21(5):739-41.

81. Tagaya Y, Matsuoka M, Gallo R. 40 years of the human T-cell leukemia virus: past, present, and future. F1000Res. 2019;8(F1000 Faculty Rev):228.

82. Khoury G, et al. Deleted HTLV-1 subtype C proviruses that retain only the $\mathrm{X}$-region persist and predominate in infected Aboriginal Central Australians. 2020. (Unpublished data).

83. Cassar $\mathrm{O}$, et al. Human T-cell lymphotropic virus type 1 subtype $\mathrm{C}$ molecular variants among indigenous australians: new insights into the molecular epidemiology of HTLV-1 in Australo-Melanesia. PLoS Negl Trop Dis. 2013;7(9):e2418.
84. Cassar O, et al. A novel human T-lymphotropic virus type 1c molecular variant in an indigenous individual from New Caledonia, Melanesia. PLoS Negl Trop Dis. 2017;11(1):e0005278.

85. Gessain A, et al. Complete nucleotide sequence of a highly divergent human T-cell leukemia (lymphotropic) virus type I (HTLV-I) variant from melanesia: genetic and phylogenetic relationship to HTLV-I strains from other geographical regions. J Virol. 1993;67(2):1015-23.

86. Galli V, et al. Essential role of human T cell leukemia virus type 1 orf-I in lethal proliferation of CD4(+) Cells in humanized mice. J Virol. 2019;93(19):e00565-19.

87. Silic-Benussi M, et al. HTLV-1 p13, a small protein with a busy agenda. Mol Aspects Med. 2010;31(5):350-8.

\section{Publisher's Note}

Springer Nature remains neutral with regard to jurisdictional claims in published maps and institutional affiliations.
Ready to submit your research? Choose BMC and benefit from:

- fast, convenient online submission

- thorough peer review by experienced researchers in your field

- rapid publication on acceptance

- support for research data, including large and complex data types

- gold Open Access which fosters wider collaboration and increased citations

- maximum visibility for your research: over $100 \mathrm{M}$ website views per year

At BMC, research is always in progress.

Learn more biomedcentral.com/submissions 